\title{
. The Rotational Spectrum of Tyrosine
}

\author{
${ }_{2}$ Cristóbal Pérez, $^{\dagger}$ Santiago Mata, Carlos Cabezas, Juan C. López, and José. L. Alonso* \\ 3 Grupo de Espectroscopia Molecular (GEM), Edificio Quifima, Laboratorios de Espectroscopia y Bioespectroscopia, Unidad Asociada \\ 4 CSIC, Parque Científico Uva, Universidad de Valladolid, 47011 Valladolid, Spain
}

\section{S Supporting Information}

6 ABSTRACT: In this work neutral tyrosine has been generated in the gas phase by laser 7 ablation of solid samples, and its most abundant conformers characterized through their 8 rotational spectra. Their identification has been made by comparison between the 9 experimental and $a b$ initio values of the rotational and quadrupole coupling constants. 10 Both conformers are stabilized by an $\mathrm{O}-\mathrm{H} \bullet \bullet \bullet \mathrm{N}$ hydrogen bond established within the 11 amino acid skeleton chain and an additional weak $\mathrm{N}-\mathrm{H} \bullet \bullet \bullet \pi$ hydrogen bond. The 12 observed conformers differ in the orientation of the phenolic $-\mathrm{OH}$ group.

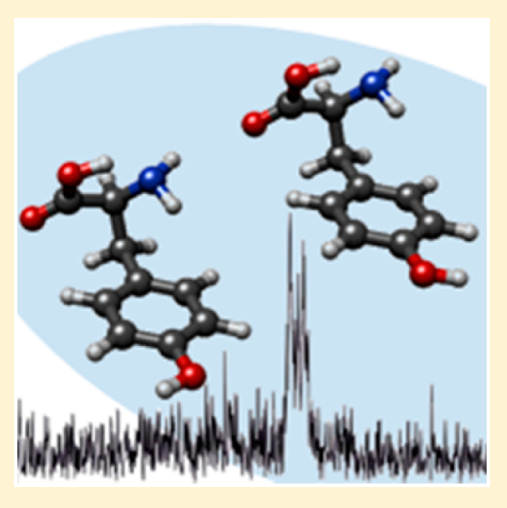

14 Proteinogenic amino acids are indispensable agents of bio15 logical function since they constitute the building blocks of 16 peptides and proteins. In addition they may have nonprotein 17 functions as neurotransmitters or being precursors of important 18 neurotransmitters or hormones. The function of proteins and 19 their multidimensional structure are highly dependent upon the 20 conformation that their constituent amino acids may adopt. 21 The knowledge of the structure and conformational behavior of 22 those building blocks is thus an important question not only to 23 biochemistry but also for chemistry, since those flexible 24 molecules constitute structural models to study the inter25 molecular forces that control molecular conformation. The 26 advantage of gas-phase conformational studies lies in the 27 opportunity to obtain the intrinsic properties of the amino acids 28 in isolation, free of the intermolecular interactions which occur 29 in condensed phases where amino acids are bipolar zwitterionic 30 species. Electronic spectroscopy techniques such as high 31 resolution laser-induced fluorescence (LIF) or resonance 32 enhanced multiphoton ionization (REMPI) in combination 33 with supersonic jets have been used to elucidate the structures 34 of gas phase proteogenic amino acids bearing chromophore 35 groups. Hence, these techniques have been applied to tyrosine 36 (Tyr), tryptophan (Trp), and phenylalanine (Phe), which were 37 the subject of a large number of investigations. ${ }^{1-18}$ REMPI and 38 LIF experiments on $\mathrm{Tyr}^{12-15}$ reported up to ten vibronic bands 39 which suggested the presence of a large number of stable 40 conformers. More recently, eight different structures of Tyr 41 were confirmed by using UV-UV and IR-UV hole burning 42 techniques. ${ }^{16,17}$ In a last work, using the same experimental 43 approach, Shimozono et al. ${ }^{18}$ interpreted the jet cooled 44 electronic spectra on the basis of 12 different conformers.
Microwave spectroscopy is particularly well adapted to the 45 study of multiconformer systems such as amino acids, since it 46 has an inherently high resolution and is exceptionally sensitive 47 to molecular geometry and mass distribution changes and it is 48 not constrained by the need for a chromophore group. 49 However, the vaporization of solid biomolecules imposed 50 serious limitations to study high melting compounds such as 51 amino acids which easily decompose by classical heating 52 methods. Recent developments in laser ablation techniques 53 have allowed us to overcome vaporization problems. In 54 particular, combining Fourier transform microwave spectros- 55 copy with laser ablation techniques in a supersonic expansion 56 (LA-MB-FTMW $)^{19-21}$ has provided a new approach to the 57 structural studies of amino acids. Apart from glycine ${ }^{22,23}$ and 58 alanine, $^{24}$ first studied by classical heating methods, a large 59 number of proteogenic and nonproteogenic aliphatic amino 60 acids $^{19-21,25-34}$ have been studied by the above technique. 61 Their applicability to aromatic amino acids was first tested in 62 phenylglycine $^{35}$ and latter applied to the studies of $\mathrm{Phe}^{36}$ and 63 Trp. ${ }^{37}$ Significant photofragmentation was detected, and 64 consequently, our experimental setup was modified trying to 65 minimize it. Shorter laser pulses (30 ps length pulse) and 66 shorter wavelengths (Nd:YAG $355 \mathrm{~nm}$ ) have been recently 67 implemented in our new instrumentation ${ }^{37,38}$ in order to 68 minimize photofragmentation processes. In the context of our 69 ongoing investigation of the conformational panorama of 70 amino acids using rotational spectroscopy, we report in this 71 paper the first rotational study of the proteogenic aromatic 72 amino acid tyrosine.

Received: February 24, 2015

Revised: March 31, 2015 
Table 1. Experimental Spectroscopic Parameters for the Observed Conformers IIa1 and IIa2 of L-Tyrosine

\begin{tabular}{|c|c|c|c|c|}
\hline \multirow[b]{2}{*}{ Parameter $^{a}$} & \multicolumn{2}{|c|}{ Experimental } & \multicolumn{2}{|c|}{ Theory } \\
\hline & Rotamer X & Rotamer Y & Conformer IIal & Conformer IIa2 \\
\hline A & $1529.6791(40)^{b}$ & $1525.2543(29)$ & 1519.5 & 1516.4 \\
\hline B & $463.94021(32)$ & $465.48173(25)$ & 472.6 & 474.2 \\
\hline $\mathrm{C}$ & $425.76168(40)$ & $427.31023(27)$ & 433.2 & 434.8 \\
\hline$\Delta_{\mathrm{J}}$ & $0.0507(29)$ & $0.0527(19)$ & & \\
\hline$\chi_{\mathrm{aa}}$ & $0.709(14)$ & $0.740(15)$ & 0.94 & 0.96 \\
\hline$\chi_{\mathrm{bb}}$ & $0.236(88)$ & $0.247(92)$ & -0.29 & -0.28 \\
\hline$\chi_{\mathrm{cc}}$ & $-0.945(88)$ & $-0.988(92)$ & -0.64 & -0.67 \\
\hline
\end{tabular}

${ }^{a} \mathrm{~A}, \mathrm{~B}$, and $\mathrm{C}$ are the rotational constants; $\Delta_{\mathrm{J}}$ is the cuartic centrifugal distortion constant; $\chi_{\mathrm{aa}} \chi_{\mathrm{bb}}$, and $\chi_{\mathrm{cc}}$ are the diagonal elements of the ${ }^{14} \mathrm{~N}$ nuclear quadrupole coupling tensor. ${ }^{b}$ Standard error in parentheses in units of the last digit.

\section{EXPERIMENTAL SECTION}

75 The rotational spectrum of Tyr was observed using a new laser 76 ablation molecular beam Fourier transform microwave (LA77 MB-FTMW $)^{20,21,38}$ spectrometer operating in the $4-10 \mathrm{GHz}$ 78 frequency range. Solid rods of fine-powdered tyrosine (mp 79 290-295 ${ }^{\circ} \mathrm{C}$ ) were mixed with minimum quantities of a 80 commercial binder to form a cylindrical rod. The samples were 81 then vaporized using the third harmonic $(355 \mathrm{~nm})$ of a $82 \mathrm{Nd}$ :YAG picosecond laser (30 ps length pulse) using energies 83 of $\sim 2 \mathrm{~mJ} /$ pulse. The neutral vaporized molecules were seeded 84 in the carrier gas ( $\mathrm{Ne}, 15$ bar) and expanded into a Fabry-Pérot 85 resonator. After sending the microwave pulses through the 86 cavity, the emission FID (free induction decay) of the 87 molecules was recorded in the time-domain and Fourier 88 transformed to yield the frequency-domain spectrum. Since the 89 supersonic jet and the microwave resonator axis are collinearly 90 placed, signals appeared split into Doppler doublets. The 91 arithmetic mean of the doublets was taken as the final 92 frequency. The estimated accuracy of the frequency measure93 ments is better than $3 \mathrm{kHz}$.

\section{RESULTS AND DISCUSSION}

95 Before starting the experimental study, we extended the 96 previous $a b$ initio calculations ${ }^{39}$ on the low energy conformers 97 of Tyr to predict the rotational and nuclear quadrupole 98 coupling constants as well as electric dipole moment 99 components which are needed for the interpretation of the 100 rotational spectrum. Geometry optimizations were carried out 101 with the Gaussian suite of programs ${ }^{40}$ using second-order 102 Møller-Plesset perturbation theory (MP2) in the frozen-core 103 approximation and Pople's 6-311++G(d,p) basis set. This level 104 of theory has proven to give very good results for the rotational 105 parameters at a reasonable computational cost. ${ }^{19-33}$ The 106 calculated spectroscopic parameters for the ten lowest-energy 107 conformers of Tyr are shown in Table S1 of the Supporting 108 Information.

109 All conformers are predicted to be near-prolate asymmetric 110 tops with a nonzero $\mu_{\mathrm{a}}$ component of the electric dipole 111 moment. In this way, their $\mathrm{R}$ - branch, $\mu_{\mathrm{a}}$-type spectra are 112 expected to show the characteristic patterns consisting of 113 groups of lines separated by approximately $B+C$. Initially, the 114 polarization power was set to optimally polarize the transitions 115 associated with moderate values of $\mu_{\mathrm{a}}$. Hence, we were able to 116 detect two sets of weak $\mu_{\mathrm{a}}$-type R-branch series transitions, 117 attributable to two different rotamers which initially were 118 labeled as $\mathrm{X}$ and $\mathrm{Y}$. The subsequent fitting and prediction 119 iterative procedure allows us to observe new $\mu_{\mathrm{a}}$ transitions and 120 to extend our measurements to the R-branch $\mu_{\mathrm{b}}$-type spectrum.
All the observed transitions (see Figure S1 and Table S3 and S4 121 of the Supporting Information) were split into several close 122 hyperfine components showing the characteristic pattern due to 123 a ${ }^{14} \mathrm{~N}$ nucleus, which confirms the presence of a single nitrogen 124 atom in the observed species. This hyperfine structure arises 125 from the interaction of the electric quadrupole moment of the 126 ${ }^{14} \mathrm{~N}(I=1)$ nucleus with the electric field gradient created at the ${ }_{127}$ site of the quadrupolar nucleus by the rest of the electronic and 128 nuclear charges of the molecule. This interaction gives rise to 129 the coupling of the ${ }^{14} \mathrm{~N}$ nuclear spin with the overall angular 130 momentum, which results in a characteristic hyperfine structure 131 observable in the rotational spectra. The associated spectro- 132 scopic parameters are the quadrupole coupling constants $\chi_{\alpha \beta} 133$ $(\alpha \beta=a, b, c)$. These are the elements of the quadrupole 134 coupling tensor $\chi$, which is related to the electric field gradient 135 tensor $\mathrm{q}$ by $\chi=e Q \mathrm{q}$, where $e Q$ is the electric quadrupole 136 moment. According to this, the spectra were analyzed ${ }^{41}$ using 137 the semirigid rotor Hamiltonian of Watson in the A reduction 138 and the $\mathrm{I}^{\mathrm{r}}$ representation $H_{R}{ }^{(\mathrm{A})}$, 2 supplemented with a term to 139 take into account the quadrupole interaction $H_{Q}{ }^{43}$ namely $H=140$ $H_{R}{ }^{(\mathrm{A})}+H_{Q}$. The analysis allowed the determination of the 141 rotational constants, the centrifugal distortion constant $\Delta_{\mathrm{J}}$, and 142 the diagonal elements of the quadrupole coupling tensor for 143 both $\mathrm{X}$ and $\mathrm{Y}$ rotamers (see Table 1).

The comparison of the experimental and predicted rotational 145 parameters in Table 1 shows that the observed rotational 146 constants are in good agreement with those predicted for 147 conformers IIa1 and IIa2 (collected also in Table 1 for 148 comparison). The same conclusion can be reached from the 149 comparison of the values of the ${ }^{14} \mathrm{~N}$ quadrupole coupling 150 constants. The experimental values are only in good agreement 151 with those predicted for conformers IIa1 and IIa2. The values 152 of the ${ }^{14} \mathrm{~N}$ quadrupole coupling constants are very sensitive to 153 the orientation of the $-\mathrm{NH}_{2}$ group with respect to the principal 154 inertial axis system so that in most cases these constants led to a 155 conclusive assignment of the observed conformers. However, in 156 this case, conformers IIa1 and IIa2 have practically the same 157 values of the ${ }^{14} \mathrm{~N}$ quadrupole coupling constants because the 158 rotation of the principal inertial axis system induced by the 159 change in the structure of the phenolic $-\mathrm{OH}$ group does not 160 contribute to significant changes in the quadrupole coupling 161 constants. Therefore, it is not possible to carry out an 162 unequivocal assignment of both observed rotamers on the 163 basis of these constants. Fortunately, the change in orientation 164 of the $-\mathrm{OH}$ group in conformers IIa1 and IIa2 causes 165 distinctive shifts in the inertial moments which are translated to 166 the rotational constants. Thus, the small shifts in the rotational 167 constants do have the key to discern between both conformers. 168 The changes in the experimental values of the rotational 169 
170 constants between rotamers $\mathrm{X}$ and $\mathrm{Y}$ are $\Delta A=\Delta A_{\mathrm{Y}}-\Delta A_{\mathrm{X}} \approx$ $171-4.4 \mathrm{MHz}, \Delta B \approx 1.5 \mathrm{MHz}$ and $\Delta C \approx 1.5 \mathrm{MHz}$. These values 172 are in good agreement with the differences between the 173 rotational constants of conformers IIa1 and IIa2; $\Delta A=\Delta A_{\text {IIa2 }}$ $174-\Delta A_{\text {IIal }} \approx-3.1 \mathrm{MHz}, \Delta B \approx 1.6 \mathrm{MHz}$, and $\Delta C \approx 1.6 \mathrm{MHz}$. 175 This fact allows the identification of rotamers $\mathrm{X}$ and $\mathrm{Y}$ as the 176 lowest lying energy conformers IIa1 and IIa2, respectively. The 177 small discrepancy found between the experimental and 178 predicted values of quadrupole coupling constants (Table 1) 179 is due to a slight variation in the actual orientation of the amino 180 group with respect to that predicted via $a b$ initio methods. 181 Hence, when the amino group rotated $\left(6^{\circ}\right.$ on the dihedral 182 angle $\angle \mathrm{HNCC}$ ) from the equilibrium value, the predicted and 183 experimental values of the nuclear quadrupole coupling 184 constants are nearly in coincidence (see Table S2).

185 In the next stage of our investigation, we focused the 186 experimental searches to detect spectral signatures of Ia and IIb 187 conformers, predicted higher in energy. We observed sets of 188 very weak $\mu_{\mathrm{a}}$-type $\mathrm{R}$-branch rotational transitions in the 189 predicted frequency intervals for conformers IIb1 and IIb2, 190 predicted to have high values of the $\mu_{\mathrm{a}}$ electric dipole moment 191 component. We were only able to observe a few lines of each 192 set with a non-well-resolved hyperfine structure; no spectro193 scopic constants could be derived. After performing wide scans 194 with different experimental conditions, no lines attributable to 195 other conformers of Tyr were observed.

196 The two observed conformers of Tyr shown in Figure 1 are 197 stabilized by an $\mathrm{O}-\mathrm{H} \cdots \mathrm{N}$ hydrogen bond with a $\mathrm{COOH}$ trans
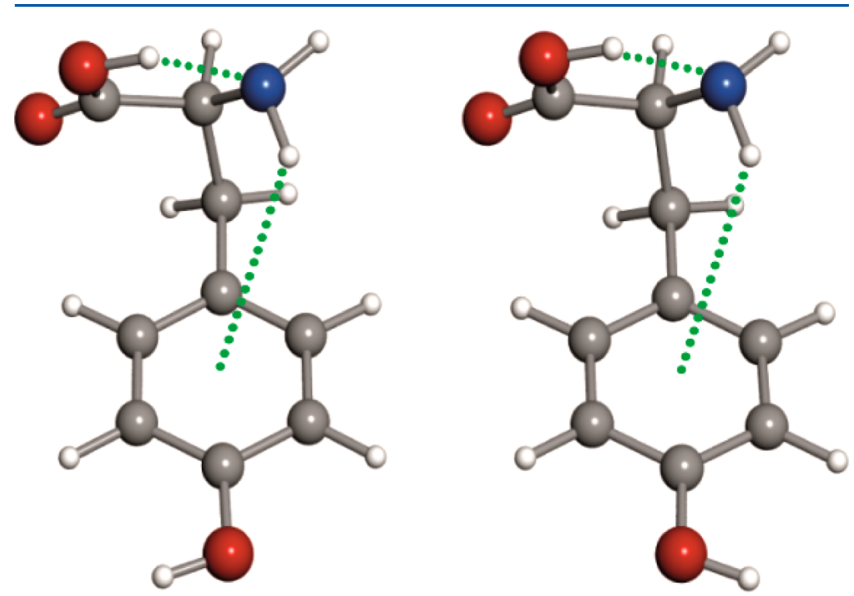

Figure 1. 3D structures of observed conformers (IIa1(left) and IIa2 (right)) of Tyr showing the intramolecular interactions which stabilize both structures.

198 configuration (type II of the amino acids ${ }^{20}$ ). Moreover, one of 199 the hydrogen atoms of the amino group is pointing toward the $200 \pi$ electron density of the ring, indicating the existence of an N$201 \mathrm{H} \cdots \pi$ interaction. The modified orientation of the amino group 202 mentioned above points to the establishment of this $\mathrm{N}-\mathrm{H} \cdots \pi$ 203 interaction by decreasing the hydrogen bond distance. Both 204 intramolecular hydrogen bonds form a chain that reinforces 205 their strength through cooperative effects. ${ }^{44,45}$ For the over206 whelming majority of the $\alpha$-amino acids studied so far, a type I 207 conformer has been found as global minimum. A few 208 exceptions have been found for which a type II conformer 209 has been found to be the most stable conformer. Those include 210 the imino acids proline, ${ }^{19,32}$ and hydroxyproline, ${ }^{28}$ asparagine, ${ }^{34}$ 211 phenylalanine, $^{36}$ tryptophan, ${ }^{37}$ and histidine. ${ }^{38}$ With the exception of proline and hydroxyproline, the type II O-H $\cdots 212$ $\mathrm{N}$ hydrogen bond established within the amino acid backbone 213 is reinforced by an additional bond from the amino group to 214 the lateral chain in all cases. In the case of the phenylalanine, ${ }^{36} 215$ tryptophan, $^{37}$ and tyrosine, this additional interaction corre- 216 sponds to a weak $\mathrm{N}-\mathrm{H} \cdots \pi$ weak hydrogen bond.

217

Relative intensity measurements on selected $\mu_{\mathrm{a}}$-type lines of 218 the IIa1 and IIa2 conformers indicate that the relative 219 populations follow the order IIa2 > IIa1, in agreement with 220 the theoretically predicted relative energies. This pair of 221 conformers, which differ only in the $\mathrm{OH}$ arrangement, have a 222 predicted energy difference of $132 \mathrm{~cm}^{-1}$. This energy difference 223 could be tentatively explained by the existence of local dipole- 224 local dipole ${ }^{46}$ interaction between the amino acid group and the 225 side chain $\left(-\mathrm{CH}_{2}-\mathrm{C}_{6} \mathrm{H}_{4}-\mathrm{OH}\right)$. These dipoles find a more 226 favorable arrangement for conformer IIa2, increasing in this 227 way the relative stability of this conformer.

In conclusion, we have observed two conformers of Tyr 229 through the analysis of its rotational spectrum. These 230 conformers are stabilized by $\mathrm{O}-\mathrm{H} \cdots \mathrm{N}$ and $\mathrm{N}-\mathrm{H} \cdots \pi$ hydrogen 231 bond interactions and derive from the most stable forms 232 observed for the related amino acid Phe. ${ }^{36}$ The weakness of the 233 spectrum and the low number of observed conformers for Tyr 234 compared with those detected for aliphatic amino acids ${ }^{30-33} 235$ can be attributable to photofragmentation/ionization processes 236 during the laser ablation course. As reported for $\mathrm{Phe},{ }^{47} 237$ ionization processes would favor the presence of only IIa and 238 IIb conformers, with higher ionization energy, in the supersonic 239 jet. We have monitored photofragmentation effects using a 240 time-of-flight mass spectrometer (TOF-MS) coupled with the 241 same laser ablation nozzle used in our LA-MB-FTMW 242 experiment and we found (See Figure S2 of the Supporting 243 Information) a large amount of Tyr fragments, indicating that 244 photofragmentation of Tyr occurs to a large extent in the 245 ablation process. The subsequent depletion of the number 246 density of tyrosine in the supersonic expansion would cause the 247 observation of weaker spectra. Despite the important improve- 248 ments recently achieved in the observation of nucleoside 249 uridine, ${ }^{48}$ the intrinsic effects of photofragmentation associated 250 with the aromatic amino acids make extremely difficult the 251 observation of their complete conformational panorama. 252

\section{ASSOCIATED CONTENT}

\section{S Supporting Information}

254

Complete ref 40, predicted ab initio molecular properties for 255 the lower-energy conformers of the tyrosine together with 256 $\mathrm{MP} 2 / 6-311++\mathrm{G}(\mathrm{d}, \mathrm{p})$ equilibrium principal axis coordinates, 257 quadrupole coupling constants calculated at the different 258 dihedral angles $\angle \mathrm{HNCC}$ for the observed conformers, 259 observed rotational transitions for the two detected conformers, 260 and list of measured transitions and mass spectrum of tyrosine. 261 This material is available free of charge via the Internet at 262 http://pubs.acs.org.

\section{AUTHOR INFORMATION}

* E-mail: jlalonso@qf.uva.es Phone: +34 983186348 Fax: +34 266 983186349.

$\dagger$ (C.P.) Max-Planck Institute for the Structure and Dynamics of 269 Matter; Luruper Chaussee 149, D-22761 Hamburg. Germany. 270 


\section{Notes}

272 The authors declare no competing financial interest.

\section{ACKNOWLEDGMENTS}

274 This research was supported by Ministerio de Ciencia e 275 Innovación (grant numbers CTQ 2010-19008, CTQ 2013276 40717-P, and Consolider Ingenio 2010 CSD 2009-00038) and 277 Junta de Castilla y León (grant number VA175U13). C.C. 278 thanks the Junta de Castilla y León for the postdoctoral 279 contract (grant number CIP13/01).

\section{$280 \quad$ REFERENCES}

281 (1) Philips, L. A.; Webb, S. P.; Martinez, S. J.; Fleming, G. R.; Levy, 282 D. H. Time-Resolved Spectroscopy of Tryptophan Conformers in a 283 Supersonic Jet. J. Am. Chem. Soc. 1988, 110, 1352-1355.

284 (2) Rizzo, T. R.; Park, Y. D.; Peteanu, L. A.; Levy, D. H. The 285 Electronic Spectrum of the Amino Acid Tryptophan in the Gas Phase. 286 J. Chem. Phys. 1986, 84, 2534-2541.

287 (3) Cable, J. R.; Tubergen, M. J.; Levy, D. H. Laser Desorption 288 Molecular Beam Spectroscopy: The Electronic Spectra of Tryptophan 289 Peptides in the Gas Phase. J. Am. Chem. Soc. 1987, 109, 6198-6199. 290 (4) Snoek, L. C.; Kroemer, R. T.; Hockridge, M. R.; Simons, J. P. 291 Conformational Landscapes of Aromatic Amino Acids in the Gas 292 Phase: Infrared and Ultraviolet Ion Dip Spectroscopy of Tryptophan. 293 Phys. Chem. Chem. Phys. 2001, 3, 1819-1826.

294 (5) Snoek, L. C.; Kroemer, R. T.; Simons, J. P. A Spectroscopic and 295 Computational Exploration of Tryptophan-Water Cluster Structures 296 in the Gas Phase. Phys. Chem. Chem. Phys. 2002, 4, 2130-2139.

297 (6) Martinez, S. J., III; Alfano, J. C.; Levy, D. H. The Electronic 298 Spectroscopy of Tyrosine and Phenylalanine Analogs in a Supersonic 299 Jet: Acidic Analogs. J. Mol. Spectrosc. 1991, 145, 100-111.

300 (7) Snoek, L. C.; Robertson, E. G.; Kroemer, R. T.; Simons, J. P. 301 Conformational Landscapes in Amino Acids: Infrared and Ultraviolet 302 Ion-Dip Spectroscopy of Phenylalanine in the Gas Phase. Chem. Phys. 303 Lett. 2000, 321, 49-56.

304 (8) Lee, K. T.; Sung, J.; Lee, K. J.; Kim, S. K.; Park, Y. D. Resonant 305 Two-Photon Ionization Study of Jet-Cooled Amino Acid: L-Phenyl306 alanine and Its Monohydrated Complex. J. Chem. Phys. 2002, 116, $3078251-8254$.

308 (9) Lee, K. T.; Sung, J.; Lee, K. J.; Park, Y. D.; Kim, S. K. 309 Conformation-Dependent Ionization Energies of L-Phenylalanine. 310 Angew. Chem., Int. Ed. 2002, 41, 4114-4117.

311 (10) Hashimoto, T.; Takasu, Y.; Yamada, Y.; Ebata, T. Anomalous 312 Conformer Dependent S1 Lifetime of L-Phenylalanine. Chem. Phys. 313 Lett. 2006, 421, 227-231.

314 (11) Ebata, T.; Hashimoto, T.; Ito, T.; Inokuchi, Y.; Altunsu, F.; 315 Brutschy, B.; Tarakeshwar, P. Hydration Profiles of Aromatic Amino 316 Acids: Conformations and Vibrations of L-phenylalanine-(H2O)n 317 Clusters. Phys. Chem. Chem. Phys. 2006, 8, 4783-4791.

318 (12) Martinez, S. J., III; Alfano, J. C.; Levy, D. H. The Electronic 319 Spectroscopy of the Amino Acids Tyrosine and Phenylalanine in a 320 Supersonic Jet. J. Mol. Spectrosc. 1992, 156, 421-430.

321 (13) Martinez, S. J.; Alfano, J. C.; Levy, D. H. The Electronic 322 Spectroscopy of Tyrosine and Phenylalanine Analogs in a Supersonic 323 Jet: Basic Analogs. J. Mol. Spectrosc. 1993, 158, 82-92.

324 (14) Lindinger, A.; Toennies, J. P.; Vilesov, A. F. High Resolution 325 Vibronic Spectra of the Amino Acids Tryptophan and Tyrosine in 0.38 326 K Cold Helium Droplets. J. Chem. Phys. 1999, 110, 1429-1436.

327 (15) Grace, L. I.; Cohen, R.; Dunn, T. M.; Lubman, D. M.; de Vries, 328 M. S. The R2PI Spectroscopy of Tyrosine: A Vibronic Analysis. J. Mol. 329 Spectrosc. 2002, 215, 204-219.

330 (16) Inokuchi, Y.; Kobayashi, Y.; Ito, T.; Ebata, T. Conformation of 331 L-Tyrosine Studied by Fluorescence-Detected UV-UV and IR-UV 332 Double-Resonance Spectroscopy. J. Phys. Chem. A 2007, 111, 32093333215.

334 (17) Abo-Riziq, A.; Grace, L.; Crews, B.; Callahan, M. P.; van 335 Mourik, T.; de Vries, M. S. Conformational Structure of Tyrosine,
Tyrosyl-glycine, and Tyrosyl-glycyl-glycine by Double Resonance 336 Spectroscopy. J. Phys. Chem. A 2011, 115, 6077-6087. 337

(18) Shimozono, Y.; Yamada, K.; Ishiuchi, S.; Tsukiyama, K.; Fujii, 338 M. Revised Conformational Assignments and Conformational 339 Evolution of Tyrosine by Laser Desorption Supersonic Jet Laser 340 Spectroscopy. Phys. Chem. Chem. Phys. 2013, 15, 5163-5175. 341

(19) Lesarri, A.; Mata, S.; Cocinero, E. J.; Blanco, S.; López, J. C.; 342 Alonso, J. L. The Structure of Neutral Proline. Angew. Chem., Int. Ed. 343 2002, 41, 4673-4676.

(20) Alonso, J. L.; Pérez, C.; Sanz, M. E.; López, J. C.; Blanco, S. 345 Seven Conformers of L-Threonine in the Gas Phase: A LA-MB- 346 FTMW Study. Phys. Chem. Chem. Phys. 2009, 11, 617-627. 347

(21) Peña, I.; Sanz, M. E.; López, J. C.; Alonso, J. L. Preferred 348 Conformers of Proteinogenic Glutamic Acid. J. Am. Chem. Soc. 2011, 349 134, 2305-2312.

(22) Brown, R. D.; Godfrey, P. D.; Storey, J. W. V.; Bassez, M.-P. 351 Microwave Spectrum and Conformation of Glycine. J. Chem. Soc., 352 Chem. Commun. 1978, 547-548.

(23) Suenram, R. D.; Lovas, F. J. Millimeter Wave Spectrum of 354 Glycine. J. Mol. Spectrosc. 1978, 72, 372-382. 355

(24) Godfrey, P. D.; Firth, S.; Hatherley, L. D.; Brown, R. D.; Pierlot, 356 A. P. Millimeter-Wave Spectroscopy of Biomolecules: Alanine. J. Am. 357 Chem. Soc. 1993, 115, 9687-9691.

(25) Blanco, S.; Lesarri, A.; López, J. C.; Alonso, J. L. The Gas-Phase 359 Structure of Alanine. J. Am. Chem. Soc. 2004, 126, 11675-11683. 360

(26) Lesarri, A.; Cocinero, E. J.; López, J. C.; Alonso, J. L. The Shape 361 of Neutral Valine. Angew. Chem., Int. Ed. 2004, 43, 605-610. 362

(27) Lesarri, A.; Sánchez, R.; Cocinero, E. J.; López, J. C.; Alonso, J. 363 L. Coded Amino Acids in Gas Phase: The Shape of Isoleucine. J. Am. 364 Chem. Soc. 2005, 127, 12952-12956.

(28) Lesarri, A.; Cocinero, E. J.; López, J. C.; Alonso, J. L. Shape of 366 4(S)- and 4(R)-Hydroxyproline in Gas Phase. J. Am. Chem. Soc. 2005, 367 $127,2572-2579$.

(29) Cocinero, E. J.; Lesarri, A.; Grabow, J.-U.; López, J. C.; Alonso, 369 J. L. The Shape of Leucine in the Gas Phase. ChemPhysChem 2007, 8, 370 599-604.

(30) Blanco, S.; Sanz, M. E.; López, J. C.; Alonso, J. L. Revealing the 372 Multiple Structures of Serine. Proc. Natl. Acad. Sci. 2007, 104, 20183- 373 20188.

(31) Sanz, M. E.; Blanco, S.; López, J. C.; Alonso, J. L. Rotational 375 Probes of Six Conformers of Neutral Cysteine. Angew. Chem., Int. Ed. 376 2008, 47, 6216-6220.

377

(32) Mata, S.; Vaquero, V.; Cabezas, C.; Peña, I.; Pérez, C.; López, J. 378 C.; Alonso, J. L. Observation of Two New Conformers of Neutral 379 Proline. Phys. Chem. Chem. Phys. 2009, 11, 4141-4144. 380

(33) Sanz, M. E.; López, J. C.; Alonso, J. L. Six Conformers of 381 Neutral Aspartic Acid Identified in the Gas Phase. Phys. Chem. Chem. 382 Phys. 2010, 12, 3573-3578.

(34) Cabezas, C.; Varela, M.; Peña, I.; Mata, S.; López, J. C.; Alonso, 384 J. L. The Conformational Locking of Asparagine. Chem. Commun. 385 2012, 48, 5934-5936.

(35) Sanz, M. E.; Cortijo, V.; Caminati, W.; López, J. C.; Alonso, J. L. 387 The Conformers of Phenylglycine. Chem.-Eur. J. 2006, 12, 2564- 388 2570.

(36) Pérez, C.; Mata, S.; Blanco, S.; López, J. C.; Alonso, J. L. Jet- 390 Cooled Rotational Spectrum of Laser-Ablated Phenylalanine. J. Phys. 391 Chem. A 2011, 115, 9653-9657.

(37) Sanz, M. E.; Cabezas, C.; Mata, S.; Alonso, J. L. Rotational 393 Spectrum of Tryptophan. J. Chem. Phys. 2014, 140, 204308.

(38) Bermúdez, C.; Mata, S.; Cabezas, C.; Alonso, J. L. Tautomerism 395 in Neutral Histidine. Angew. Chem., Int. Ed. 2014, 53, 11015-11018. 396 (39) Zhang, M.; Huang, Z.; Lin, Z. Systematic Ab Initio Studies of 397 the Conformers and Conformational Distribution of Gas-Phase 398 Tyrosine. J. Chem. Phys. 2005, 122, 134313.

(40) Frisch, M. J.; Trucks, G. W.; Schlegel, H. B.; Scuseria, G. E.; 400 Robb, M. A.; Cheeseman, J. R.; Montgomery, J. A., Jr.; Vreven, T.; 401 Kudin, K. N.; Burant, J. C.; et al. GAUSSIAN 03 (Revision B.04) (See 402 Supporting Information for full citation).

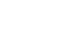

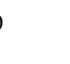
.

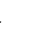

\section{.}

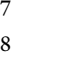

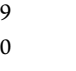
(n)

(n)


404 (41) Pickett, H. M. The Fitting and Prediction of Vibration-Rotation 405 Spectra with Spin Interactions. J. Mol. Spectrosc. 1991, 148, 371-377. 406 (42) Watson, J. K. G. In Vibrational Spectra and Structure; Durig, J. R., 407 Ed.; Elsevier: New York, 1977; Vol. 6, pp 1- 78.

408 (43) Gordy, W.; Cook, R. L. Microwave Molecular Spectra, 3rd ed.; 409 Wiley: New York, 1984.

410 (44) Saenger, W. Nature (London) 1979, 279, 343.

411 (45) Jeffrey, G. A. An Introduction to Hydrogen Bonding; Oxford 412 University Press: New York, 1997.

413 (46) The Weak Hydrogen Bond in Structural Chemistry and Biology; 414 IUCr Monographs on Crystallography, Vol. IX; Desiraju, G. R., 415 Steiner, T., Eds.; Oxford University Press: Oxford, 2001.

416 (47) Lee, K. T.; Sung, J.; Lee, K. J.; Park, Y. D.; Kim, S. K. 417 Conformation-dependent ionization energies of L-phenylalanine. 418 Angew Chem., Int. Ed. 2002, 41, 4114-4117.

419 (48) Peña, I.; Cabezas, C.; Alonso, J. L. The Nucleoside Uridine 420 Isolated in the Gas Phase. Angew. Chem., Int. Ed. 2015, 10, 2991-2994. 